\title{
BENCHMARKING OF CODES FOR CALCULATING LOCAL NET EC POWER LOSSES IN FUSION PLASMAS
}

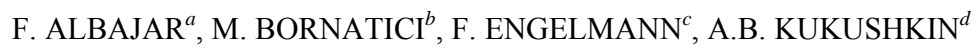 \\ ${ }^{a}$ Asociación EURATOM-CIEMAT, Madrid, 28040, Spain \\ ${ }^{b}$ Physics Department “A. Volta”, University of Pavia, Pavia, 27100, Italy \\ ${ }^{\mathrm{c}}$ Max-Planck-Institut für Plasmaphysik, Garching, 85748, Germany \\ ${ }^{\mathrm{d}}$ NFI RRC “Kurchatov Institute”, Moscow, 123182, Russia
}

\begin{abstract}
The codes SNECTR, CYTRAN, CYNEQ and EXACTEC are compared in view of the calculation of the profile of the net electron cyclotron (EC) wave power density emitted for different electron temperature profiles and average temperatures of relevance for reactor-grade magnetoplasmas. The effects of either specularly or diffusely reflecting walls are assessed for a cylindrical plasma with circular cross-section, specular reflection, as assumed in EXACTEC, providing a lower bound to the net EC wave power losses in the hot plasma core (and therefore, as a rule, also to the total EC power loss) as well as to re-absorption in the edge plasma. The assumption of isotropy of the radiation intensity in the plasma underlying both CYTRAN and CYNEQ, is discussed and found to be adequate for strong diffuse reflection, however overestimating the net EC loss in the plasma core for weakly reflecting walls by up to $20 \%$. On the whole, the full transport code SNECTR (no longer in active use), for specular reflection, and the exact cylindrical code EXACTEC are in excellent agreement with each other, both CYTRAN and CYNEQ resulting to be fast routines suited for use in systematic transport simulations of fusion plasmas.
\end{abstract}

\section{Introduction}

In view of the potential importance of electron cyclotron (EC) wave emission in the local electron power balance for ITER and tokamak reactor steady-state operation [1], fast routines are needed for use in systematic transport simulations of fusion plasmas. The code SNECTR developed by Tamor [2] provides a solution of the radiative transport equation (RTE) in an arbitrary axisymmetric magnetoplasma with (either specular or diffuse) reflecting walls, for specified density, temperature and magnetic field profiles, assuming the electron distribution function to be locally Maxwellian. Soon after, Tamor developed the simple fast routine CYTRAN [3] which contains the main physics of SNECTR, a distinctive basic assumption being that the radiation field in the plasma is taken to be isotropic (which suppresses all effects due to an angular dependence of the radiation field). Somewhat similar to CYTRAN is the 
CYNEQ code, the latter allowing, however, for an arbitrary isotropic electron distribution function [4]. Common to both CYTRAN and CYNEQ is the division of the plasma in an optically thick inner region and an optically thin outer region, the thick-thin interface $r_{\text {thin-thick }}$ being determined in terms of a critical optical thickness along the radial coordinate $r$,

$$
\tau_{\text {crit }}^{\sigma}\left(r_{\text {thin-thick }}, \omega\right)=\int_{r_{\text {rinin-thick }}}^{r_{\text {dege }}} d r^{\prime}\left\langle\alpha^{\sigma}\left(r^{\prime}, \omega, \hat{\mathbf{s}}\right)\right\rangle,
$$

where $\left\langle\alpha^{\sigma}>\right.$ is the absorption coefficient averaged over the angle of propagation with $\hat{\mathbf{s}}$ referring to the ray direction, $\sigma$ denotes the wave mode and $\omega$ is the wave frequency. The value of the critical optical thickness is fixed according to results obtained from the more exact Monte-Carlo simulations using SNECTR.

More recently, based on an exact analytical solution of the RTE for a (circularly) cylindrical Maxwellian plasma with specularly reflecting walls, the code EXACTEC has been developed and used for calculating local net EC power losses in fusion plasmas [5]. Note that in this case the radiation reflected at the wall back into the plasma is most strongly concentrated in the hot core and, hence, its re-absorption is maximised. Therefore, this case is expected to minimise the net EC wave power losses from the core plasma (as well as the total EC power loss) in comparison to other wall reflection properties and wall geometries (cross-section, toroidicity).

In this paper benchmarking results in circularly cylindrical geometry for the codes SNECTR, CYTRAN, CYNEQ and EXACTEC are reported, referring to calculations of the profile of the net EC wave power density emitted, $d P / d V$, for different electron temperature profiles and average temperatures of relevance for reactor-grade plasmas.

\section{Discussion of the results of the code benchmarking}

The code benchmarking has been carried out assuming a radial profile of the form

$$
X(\rho)=X(1)+[X(0)-X(1)]\left(1-\rho^{\beta_{X}}\right)^{\gamma_{X}}, \quad \rho=r / a,
$$

for both the electron density, $X=n_{\mathrm{e}}$, and the electron temperature, $X=T_{\mathrm{e}}$, with $X(0)$ and $X(1)$ denoting the corresponding values at the centre and at the edge of the plasma, respectively ( $a$ is the radius of the cylindrical plasma). The volume average temperature then is given by 


$$
\left\langle T_{e}\right\rangle=T_{e}(1)+\frac{2 \Gamma\left(2 / \beta_{T_{e}}\right) \Gamma\left(1+\gamma_{T_{e}}\right)}{\beta_{T_{e}} \Gamma\left(1+\gamma_{T_{e}}+2 / \beta_{T_{e}}\right)}\left[T_{e}(0)-T_{e}(1)\right]
$$

$\Gamma(X)$ being the gamma function. The magnetic field is taken to be uniform.

Let us examine first two opposite limiting cases, namely, the case of no wall reflection, shown in Fig.1(a), and a case of highly reflecting walls, $R_{\mathrm{w}}=0.98$, shown in Fig.1(b). With reference to Fig.1(a), it appears that (i) there is an excellent agreement between SNECTR and EXACTEC; (ii) both CYTRAN and CYNEQ overestimate the net radiated power density in the plasma core by up to almost $20 \%$ as a consequence of the assumption of isotropy of the radiation intensity in the plasma underlying both these codes, actually not appropriate for $R_{\mathrm{W}}=0$; (iii) CYNEQ yields a weakly nonmonotonous (plateau-like) profile adjacent to the plasma centre, in contrast to the overall monotonously decreasing profiles provided by the other three codes; (iv) CYNEQ exhibits an oscillatory-type behavior around $\mathrm{r} / a=0.7$; (v) CYNEQ tends to evidence a reversal of the $d P / d V$ profile in the cool edge plasma not obtained from the other codes.
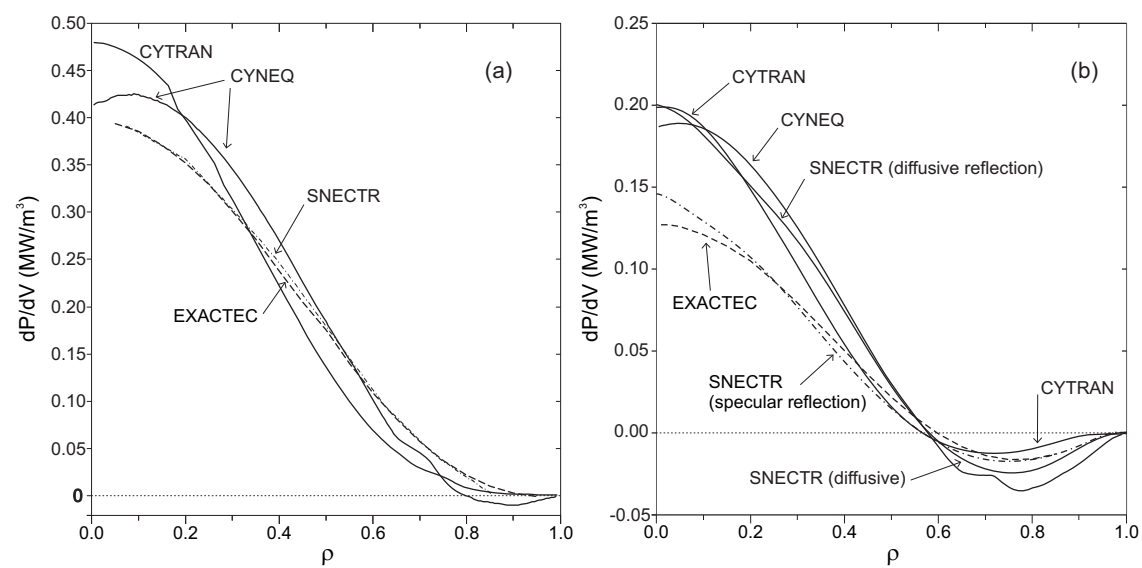

Figure 1. Radial profile of the net EC power density $d P / d V$ as given by EXACTEC, CYTRAN, CYNEQ and SNECTR for central density and temperature $n_{\mathrm{e}}(0)=10^{20} \mathrm{~m}^{-3}, T_{\mathrm{e}}(0)=50 \mathrm{keV}$, respectively, while $n_{\mathrm{e}}(1)$ and $T_{\mathrm{e}}(1)$ are 0 ; the profile indices are $\beta_{n e}=\beta_{T e}=2, \gamma_{\mathrm{ne}}=0.5$ and $\gamma_{T e}=1$, the corresponding average temperature is $\left\langle T_{\mathrm{e}}>=T_{\mathrm{e}}(0) / 2=25 \mathrm{keV}\right.$. The (homogenous) magnetic field is $5 \mathrm{~T}$ and the plasma minor radius $a$ is $1.77 \mathrm{~m}$. No wall reflection $\left(R_{\mathrm{w}}=0\right.$; left plot (a)), strong reflection $\left(R_{\mathrm{w}}=0.98\right.$; right plot (b)).

Turning now to the case of highly reflecting walls, shown in Fig.1(b), one sees that (i) SNECTR with specular reflection and EXACTEC again agree well with each other, the profile produced by SNECTR being somewhat more peaked 
(by $10 \%$ typically) towards the plasma centre; (ii) SNECTR with diffuse reflection, CYTRAN and CYNEQ are in good agreement with each other which for the strong wall reflection considered $\left(R_{\mathrm{w}}=0.98\right)$ validates the assumption of isotropy of the radiation field inherent in both CYTRAN and CYNEQ; (iii) as expected, the case of specular reflection provides a lower bound to the net power losses in the hot plasma core; (iv) CYNEQ yields the same features as mentioned in points (iii) and (iv) of the comments to Fig.1(a) are present in Fig.1(b); (v) a reversal of the $d P / d V$ profile adjacent to the plasma edge is found by all four codes, CYNEQ yielding the largest effect and SNECTR results documenting that isotropisation, as to be expected, tend to increase reabsorption of reflected radiation in the edge plasma; (vi) with respect to the case $R_{\mathrm{w}}=0$, see Fig.1(a), the net power losses in the plasma core are typically reduced by a factor 2 .
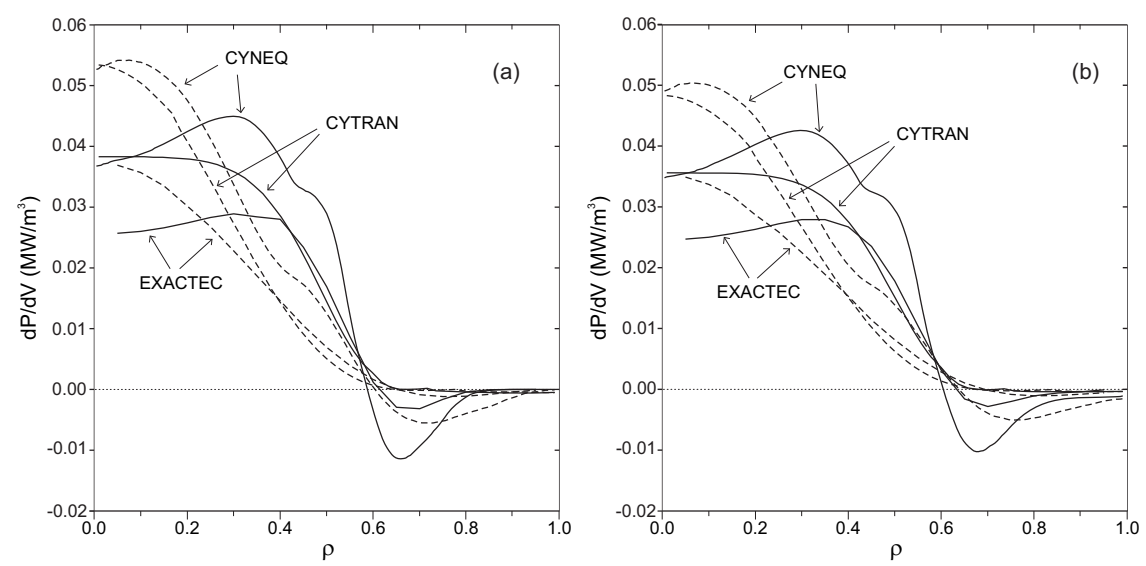

Figure 2. Radial profile of $d P / d V$ as obtained from EXACTEC, CYTRAN and CYNEQ with density given by $n_{\mathrm{e}}(0)=1.1 \times 10^{20} \mathrm{~m}^{-3}, \beta_{\mathrm{ne}}=2$ and $\gamma_{\mathrm{ne}}=0.1$; for both an "advanced" temperature profile (solid line) with $\beta_{\mathrm{Te}}=5.4, \gamma_{\mathrm{Te}}=8$, and a parabola-type one (dashed line) with $\beta_{\mathrm{Te}}=2, \gamma_{\mathrm{Te}}=1.5$; for both profiles $T_{\mathrm{e}}(0)=22.5 \mathrm{keV}$, the edge parameters: $n_{\mathrm{e}}(1)=0$ and $T_{\mathrm{e}}(1)=0.01 \mathrm{keV}$ (left plot (a)), and $n_{\mathrm{e}}(1)=0.5 \times 10^{20} \mathrm{~m}^{-3}$ and $T_{\mathrm{e}}(1)=2 \mathrm{keV}$ (right plot (b)), the corresponding average temperatures being $\left\langle T_{\mathrm{e}}>=9 \mathrm{keV}\right.$ and $10.2 \mathrm{keV}$, respectively. The magnetic field is $5.3 \mathrm{~T}$ and the plasma minor radius $a$ is $2.0 \mathrm{~m}$. The wall reflection coefficient is $R_{\mathrm{w}}=0.8$.

The more realistic case of a wall reflection coefficient $R_{\mathrm{w}}=0.8$ (for which, however, for the cylindrical geometry considered, no SNECTR results appear to be available in the literature) has been considered for three different temperature profiles, namely, an "advanced" and a parabola-type profile (having the same peak and average temperatures), see Figs.2 and 3, and a peaked profile, see Fig.4. With reference to the former figures, a distinctive feature of the advanced 
$T_{\mathrm{e}}$ profile (solid curves) is a plateau-like behaviour of $d P / d V$ in the plasma core, i.e., for $0 \leq(\mathrm{r} / a) \leq 0.4$, implying that the $d P / d V$ profile here mimics the $T_{\mathrm{e}}$ profile; on the other hand, for the parabolic-type $T_{\mathrm{e}}$ profile (dashed curves), the corresponding $d P / d V$ profile is monotonously decreasing. Analogously to Fig.1(b), EXACTEC again sets a lower bound to $d P / d V$ in the hot plasma core, whereas CYTRAN and even more CYNEQ yield higher net EC losses in the plasma core (by up to $20 \%$, typically). Overall, the agreement of the three codes is somewhat less good than the one exhibited in Figs.1. In particular, there is a strong tendency of CYNEQ to generate a non-monotonous $d P / d V$ profile in the hot plasma core for an advanced $T_{e}$ profile while CYTRAN minimises this effect; similarly, CYTRAN generally yields the weakest profile reversal of $d P / d V$ adjacent to the edge whereas CYNEQ produces the strongest one.
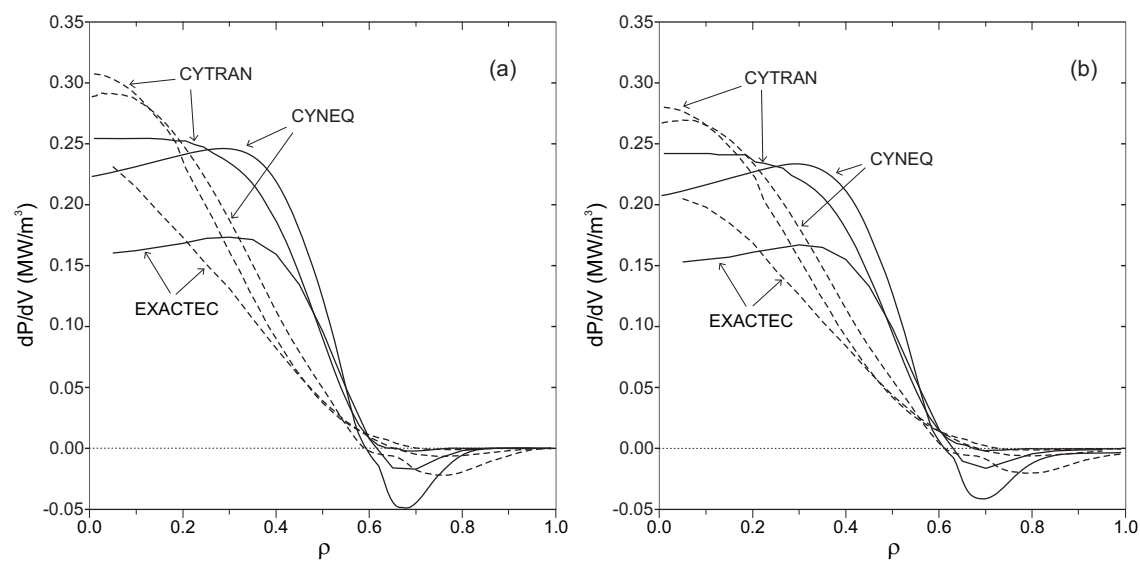

Figure 3. Same as Fig.2, but for central temperature $T_{\mathrm{e}}(0)=45 \mathrm{keV}$; edge parameters; $n_{e}(1)=0$ and $T_{e}(1)=0.01 \mathrm{keV}$ (left plot (a)), and $\mathrm{n}_{\mathrm{e}}(1)=0.5 \times 10^{20} \mathrm{~m}^{-3}$ and $\mathrm{T}_{\mathrm{e}}(1)=3 \mathrm{keV}$ (right plot (b)); the corresponding average temperatures are $\left\langle T_{\mathrm{e}}>\approx 18 \mathrm{keV}\right.$ and $\langle\mathrm{Te}>\approx 19.8 \mathrm{keV}$, respectively.

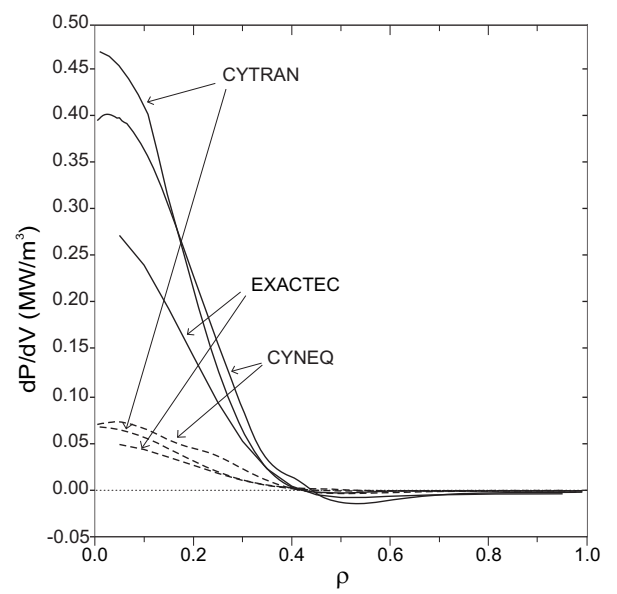

Figure 4: Radial profile of $d P / d V$ for a peaked temperature profile, $\beta_{T \mathrm{e}}=2, \gamma_{\mathrm{Te}}=5$, for the peak and edge plasma parameters of Fig.2(b), yielding an average temperature $<T_{\mathrm{e}}>\approx 5.42 \mathrm{keV}$ (dashed line), and those of Fig.3(b), corresponding to an average temperature $<T_{\mathrm{e}}>=10 \mathrm{keV}$ (solid line). 
For the case of a peaked profile, shown in Fig.4, all codes show that the net EC power losses occur mainly in the inner $10 \%$ of the plasma volume. While for $T_{\mathrm{e}}(0)=45 \mathrm{keV}$ CYTRAN, comparatively, yields the largest values of $d P / d V$ in the core plasma, for $T_{\mathrm{e}}(0)=22.5 \mathrm{keV}$ CYNEQ broadly yields the highest values of $d P / d V$.

\section{Conclusions}

Adopting a circularly cylindrical geometry, the benchmarking of the codes SNECTR, CYTRAN, CYNEQ and EXACTEC, performed in view of the calculation of the profile of the net radiated EC power density for hot fusion plasmas, shows that (i) the full transport code SNECTR, for specular wall reflection, and the (comparatively fast) exact (circularly) cylindrical code EXACTEC are in excellent agreement with each other; (ii) EXACTEC effectively provides a lower bound to the net EC wave power emitted from the hot plasma core as well as to re-absorption in the edge plasma; (iii) both CYTRAN and CYNEQ, for which the assumption of isotropy of the radiation intensity in the plasma is a distinctive feature, turn out to be fast routines wellsuited for use in systematic transport simulations of fusion plasmas.

\section{Acknowledgments}

The work by A. B. Kukushkin was supported by the Cariplo Foundation (the Landau Network-Centro Volta).

\section{References}

1. F. Albajar et al., "Importance of Electron Cyclotron Wave Energy Transport in ITER", Nucl. Fusion 45, 642 (2005).

2. S. Tamor, "Calculation of Energy Transport by Cyclotron Radiation in Fusion Plasmas," Nucl. Technol. Fusion 3, 293 (1983).

3. S. Tamor, "A simple fast routine for computation of energy transport by synchrotron radiation in tokamaks and similar geometries," Report SAI023-81-189-LJ/LAPS-72 (La Jolla, CA: Science Applications), 1981; the version used here was provided to us by W. Houlberg.

4. A.B. Kukushkin, "Heat Transport by Cyclotron Waves in Plasmas with Strong Magnetic Field and Highly Reflecting Walls," Proc. 14th IAEA Conf. on Plasma Phys. and Contr. Nucl. Fusion, 1992, v.2, pp. 35-45.

5. F. Albajar, M. Bornatici and Engelmann, "Electron Cyclotron Radiative Transfer in Fusion Plasmas," Nucl. Fusion 42, 670 (2002). 